\title{
Algunas Citas ReCIENTES DE ESPECIES DEL GÉnero Phoma Sacc. en Catalunya
}

\author{
Martí NADAL PUIGDEFABREGAS \& Asunción MORET BENASET
}

\begin{abstract}
RESUMEN: Se citan 14 táxones del género Phoma (Ph. cava, Ph. epicoccina, Ph. exigua, Ph. exigua var. foveata, Ph. glomerata, $P$ h. hedericola, $P h$. herbarum, $P h$. jolyana, $P h$. leveillei, $P h$. lycopersici, Ph. macrostoma, Ph. medicaginis var. pinodella, Ph. pomorum y $P h$. tracheiphila). Para cada uno de los táxones se indica la fecha de recolección y la especie vegetal sobre la cual se desarrollaba.
\end{abstract}

Palabras clave: Phoma, Coelomycetes.

RESUME: On cite 14 taxons du genre Phoma (Ph. cava, Ph. epicoccina, Ph. exigua, Ph. exigua var. foveata, $P$ h. glomerata, $P$ h. hedericola, $P h$. herbarum, $P h$. jolyana, $P$ h. leveillei, Ph. lycopersici, Ph. macrostroma, Ph. medicaginis var. pinodella, Ph. pomorum et $P h$. tracheiphila). Pour chacun des taxons on signale le date de racolte et l'espèce végétal sur laquelle il se develope.

Mots clés: Phoma, Coelomycetes

\section{INTRODUCCIÓN}

Una práctica habitual por parte de los antiguos micólogos era considerar que las diversas especies de hospedantes de un género de hongos eran portadoras de especies distintas de dichos patógenos. Este modo de pensar y de proceder favoreció sin duda la aparición de grandes géneros con especies poco definidas cuyo carácter distintivo principal era a menudo el desarrollarse sobre un determinado hospedante, a la vez que presentaban pequeñas diferencias morfológicas o de pigmentación que actualmente se consideran carentes de valor sistemático, puesto que, como se ha demostrado en repetidas ocasiones, estos caracteres cambian en función de la composición del medio de cultivo, relación $\mathrm{C} / \mathrm{N}$ del sustrato, intensidad de la luz, fotoperíodo, etc. El género Phoma no escapa a estas circunstancias y en consecuencia, como la mayoría de los grandes géneros, se han descrito más de 2000 especies (Sutton, 1980), plantea serios problemas la identificación de especies y táxones inferiores. 
No es de extrañar, pues, que sean abundantes en Catalunya las citas de nuevas localidades e, incluso, de nuevas especies desde principios del 1.900 (González Fragoso, 1926) que posteriormente no han podido ser refrendadas como tales. Por estos motivos consideramos que es imprescindible hacer una revisión en profundidad de las especies de este género y considerar únicamente como válidas las citas recientes que se han hecho con criterios sistemáticos actuales.

El objetivo de este trabajo es iniciar la elaboración de un catálogo con criterios sistemáticos actuales, de las especies del género Phoma existentes en Catalunya, para dar también una idea de su distribución geográfica.

\section{MATERIAL Y MÉTODOS}

Una parte considerable del material objeto de estudio fue aportado por el «Servei de Protecció dels Vegetals del Departament d’Agricultura, Ramaderia i Pesca de la Generalitat de Catalunya».

Entre los hospedantes se encuentran tanto especies espontáneas como cultivadas, pero hay que tener en cuenta que algunos ejemplares de las especies espontáneas citadas proceden de cultivo (zonas ajardinadas) y en algunas ocasiones incluso han recibido tratamientos especiales (bonsais) que han modificado su forma normal de desarrollo. En consecuencia, hay que considerar siempre las localidades que se citan como propias del hongo y no siempre como propias del hospedante.

Las determinaciones se hicieron siempre analizando material vegetal fresco a partir del cual se realizaron los aislamientos. La obtención de los cultivos puros es imprescindible para reducir al máximo la variabilidad de caracteres debida a diferencias nutricionales y factores ambientales; se utilizó como medio de cultivo standard AgarMalta, manteniéndose los cultivos a $20-22^{\circ} \mathrm{C}$, a oscuras durante la primera semana para determinar la tasa de crecimiento y luego se alternaron períodos de oscuridad y de iluminación, con luz negra (próxima al ultravioleta) de 12 h. Para la determinación de las especies se utilizaron las claves sistemáticas de Sutton, Brian (1980) basadas en las Boerema \& al.

\section{RESULTADOS}

Se citan 14 táxones del género Phoma determinados sobre muestras vegetales procedentes de diversas comarcas catalanas. Aunque no ha sido posible recolectar material vegetal de todos los municipios de Catalunya sí que se muestrearon muchos más que los citados, aunque con resultado negativo. Por otra parte, se tomaron muestras de localidades bastante distantes entre sí, tanto de la zona costera como del interior.

Para cada una de las especies aisladas se da una breve descripción y se indican la o las localidades, hospedantes y fecha de recolección de la muestra. Hemos 
prescindido de las citas que tienen en común la localidad y el hospedante con fechas de recolección próximas, sin embargo las hemos mantenido cuando las variedades del hospedante son distintas puesto que esta información puede ser útil.

No incluimos en esta relación Ph. mammillariae Ell. et Ev.; (Nadal \& Moret, 1982) por haberse determinado con criterios taxonómicos distintos de los actuales.

Phoma cava Schulz. Verh. Zool.-Bot. Ges. Wien. 21: 1248 (1871).

Forma colonias de color gris oliváceo, con denso micelio aéreo aterciopelado, sin anillos de crecimiento concéntricos; por el reverso es de color azafrán a albaricoque. Presenta células conidiógenas integradas, sobre conidióforos tabicados; los lóculos conidiógenos se forman inmediatamente por debajo del tabique transversal. Los conidios de 3-4 x 1-2 $\mu \mathrm{m}$ son rectos o ligeramente curvados, cilíndricos o ligeramente irregulares y en ocasiones gutulados. Citado (Sutton, 1980) en hojas de Hedera helix L. en Holanda y New York (USA).

Recolectado: en St. Ferriol (01-X-88) sobre hojas de Hedera helix L.

Phoma epicoccina Punithalingam, Tulloch et Leach, Trans. Br. mycol. Soc. 59: 341 (1972).

Forma colonias de pigmentación extraordinariamente variable (presentando una gama parecida a la de Epicoccum purpurascens); micelio aéreo presente, con zonación o sin ella. Los conidios de 5-8,5 x 2,5-3 $\mu \mathrm{m}$, no gutulados o bien presentan pequeñas gútulas, son rectos o ligeramente curvados, y cilíndricos; suelen estar presentes también los conidios de la forma Epicoccum purpurascens de 15-25 $\mu \mathrm{m}$ de diámetro, los cuales son muriformes y verrugosos.

Aparece citada (Sutton, 1980) en semillas de Dactylis glomerata L., Avena sp., y en Beta vulgaris L., Pyrus sp., Triticum sp., Zea mays L. La forma Epicoccum purpurascens se considera polífaga, muy común y cosmopolita (Ellis, 1971).

Recolectado: en St. Joan Despí (28-V-85) sobre Triticum aestivum L. y en St. Pere Pescador (29-IV-86) sobre ramas de MaPyrus malus L.

Phoma exigua Desm., ann. Sci. Nat. Ser. 3, 11: 282 (1849)

Forma colonias muy variables con los márgenes lobulados, generalmente aterciopeladas, con el micelio aéreo blanco, de color castaño oliváceo o incluso negro. Aplicando hidróxido sódico el agar toma color verde azulado y pasa luego a rojo castaño. Los conidios son rectos o ligeramente curvados, elipsoidales o cilíndricos, con frecuencia bigutulados y cuando maduros uniseptados, de 5,5-10 x 2,5-3,5 $\mu \mathrm{m}$.

Esta especie está citada en hospedantes de 74 géneros distintos y su distribución geográfica es bastante amplia.

Recolectado siempre sobre Solanum tuberosum L. procedente de Blanes (27-VII-85); Cambrils (03-III-88); Riudoms (07-VIII-88) y La Seu d'Urgel (30-VII-87).

La determinación de la variedad con el cultivo de El Brull (06-X-87) permitió identificarla como Ph. exigua Desm. var. foveata (Foister) Boerema la cual se distingue porque los pigmentos difundidos al medio son de color marrón amarillento y con la adición de $\mathrm{NaOH}$ el agar toma color azul grisáceo, el cual pasa luego a marrón rojizo. En los cultivos viejos se pueden observar cristales de color amarillo verdoso.

Phoma glomerata (Cda.) Wollen, et Hochapf. Z. Parasit-kde 8: 592 (1936).

Produce micelio aéreo escaso, sin embargo algunas cepas producen sectores de colonias con micelio aéreo denso, de color gris oliváceo, blanquecino o castaño. Los conidios son cilíndricos de 5-9 x 2,5-3 $\mu \mathrm{m}$, rectos o a menudo ligeramente curvados, con los extremos 
redondeados o truncados, generalmente bigutulados. Las clamidósporas, de color castaño, se forman en cadenas y presentan tabiques longitudinales y transversales; por su aspecto recuerdan los conidios de Alternaria sp.

Las colonias son poco características ya que su aspecto y coloración varía según la relación $\mathrm{C} / \mathrm{N}$ del sustrato y edad del cultivo. La relación antes indicada influye en la formación de picnidios, tamaño de las clamidosporas, etc.

Se ha citado en especies de 94 géneros de hospedantes, así como en el suelo, papel, pinturas, etc. Su distribución geográfica es muy amplia, pero hasta la fecha, en la Península Ibérica sólo aparece citado en Portugal. Nosotros aislamos esta especie en muestras de Ulmus minor Miller, Abrera (31-V-85); Cupressus macrocarpa Hertw. Alella (02-X-87); Pyrus communis L., Fontanilles (06-VI-82), Pals (12-X-82), Torroella de Montgrí (12-X-82); Corylus avellana L. El Milà (25-VII-86) y Pyrus malus L., Vilaseca i Salou (20-IV-82).

Phoma hedericola (Dur. et Mont.) Boerema, Trans. Br. Mycol. Soc. 67:295 (1976).

Forma colonias de margen regular y color negro-verdoso u oliváceo, con el micelio aéreo denso y corto. $\mathrm{Al}$ añadir $\mathrm{NaOH}$ al cultivo, el agar toma color azul-verde en muchos aislamientos. Los conidios son elipsoidales y no gutulados, generalmente de 3,5-5 x 1,25-2,5 $\mu \mathrm{m}$ en medio de cultivo, pero los desarrollados en el hospedante suelen ser algo mayores (4$6,5 \times 2,5-3,5 \mu \mathrm{m})$.

Citada en hojas y tallos de Hedera helix y H. himalaica en Austria, Checoslovaquia, India, Inglaterra, Italia, Malta, Pakistán y Portugal.

- Recolectada siempre sobre Hedera helix L. en Abella de la Conca (01-III-80), L'Ametlla del Vallès (08-XI-88), Barcelona (25-I-80), Cabrils (14-VII-87). El Masnou (19-IV-80), St. Cugat del Vallès (17-II-80), St. Just Desvern (04-VI-87), St. Pere de Ribes (07-V-88), Subirats (29-V-88).

Phoma herbarum Wested. Bull. Acad. Belg. Cl. Sci. 19: 118 (1852).

Forma colonias de crecimiento lento, con micelio aéreo escaso, de color gris-verde, sin embargo a veces en algunos sectores o incluso en toda la colonia en algunas cepas, el desarrollo del micelio aéreo es abundante pero prácticamente faltan los picnidios, tan abundantes en las cepas ralas. La pigmentación es típicamente rojiza, pero aplicando $\mathrm{NaOH}$ se vuelve azul. Los conidios son cilíndricos y rectos o elipsoidales, no gutulados, de 4-5 x 1,5-2 $\mu \mathrm{m}$.

Ha sido citado en especies de 35 géneros de hospedantes, en el aire, en el suelo, etc. Ellis, M.B. \& Ellis, J.P., 1985 considera que esta especie es frecuente en tallos herbáceos muertos, particularmente durante la primavera. Su distribución geográfica es muy amplia pero en Europa sólo aparece citado en Alemania, Checoslovaquia, Chipre, Holanda, Inglaterra, Irlanda, Yugoslavia. En España fue citado por Losa, 1948.

Recolectado en: Parlava sobre Hordeum vulgare L. (27-III-87).

Phoma jolyana Pirozynski et Morgan-Jones. Trans. Br. Mycol. Soc. 51: 200 (June, 1968).

Forma colonias variables, de color negro verdoso, con o sin micelio aéreo fieltroso; en cultivos iluminados, ocasionalmente se producen sectores con cepas más oscuras. Las clamidosporas, con aspecto de conidios de Alternaria, generalmente se desarrollan aisladas en los ápices de las hifas o bien en posición lateral; picnidios abundantes y conidios elipsoidales o ligeramente irregulares y a menudo bigutulados, de 6-7,5 x 3-4 $\mu \mathrm{m}$. Citada (Sutton, 1980) en Citrus sp. Coffea arabica, Eriobotrya japonica, Musa sp., Sterculia urens, en el suelo y en el aire en la India y Kenia. 
Recolectada en Tortosa sobre Citrus aurantium L. (28-V-87) y en Vila Seca i Salou sobre Citrus nobilis (25-V-87) y Corylus avellana (14-IV-87).

Phoma leveillei Boerema et Bollen Persoonia, 8: 115 (1975).

Colonias de color gris castaño de crecimiento lento, con o sin micelio aéreo fieltroso que presenta zonación concéntrica. Picnidios abundantes en cultivos no fieltrosos. Conidios cilíndricos, rectos y bigutulados de 2,5-3,5 x 1,5 $\mu \mathrm{m}$.

Su distribución geográfica es muy amplia, comprende Canadá, Egipto, Gana, India, Inglaterra, Kuwait, New Zeland, Omán, Singapore, Sudán y Yugoslavia y ha sido citado en Capsicum annuum, Chrysanthemum sp., Coffea arabica, Combretum sp., Fragaria vesca, Hevea sp., Juniperus depressa, Pyrus sp., Pothos sp., Rhodondendron sp., Scabiosa ochroleuca, Vaucheria filamentosa, suelo y aguas dulces.

Recolectado en: Alguaire y Bellvís sobre Pyrus malus L. el 27-XII-85 y el 12-VII-84 respectivamente; en Calella sobre Fragaria vesca L. x Fragaria chiloensis D.; en Lleida sobre Brassica oleracea (L.). (23-I-89) y sobre Pyrus malus L. (22-V-84); en Miralcamp sobre Pyrus malus L., P. malus L. cv. golden y P. malus L. cv. starking el 31-XII-85; en Premià de Dalt sobre Juniperus sp., (17-X-88) y Rhododendron japonicum (A. Gray) Sw. (10-IV-86); en Sta. Susana sobre Capsicum frutescens L. (30-VIII-88) y Fragaria vesca L. x Fr. chiloensis D. (03-V-89) y en Tordera sobre Actinidia sinensis (02-X-87).

Phoma lycopersici Cke., Grevillea, 13: 94 (1885).

Forma colonias de color gris oliváceo con micelio aéreo fieltroso denso a menudo agrupado en haces estilboides; sin zonación concéntrica; reverso de la colonia de color marrón oscuro; picnidios escasos. Los conidios con frecuencia presentan un septo mediano, son variablemente gutulados pero generalmente tienen una gútula en cada extremo, rectos, cilíndricos o elipsoidales, de 4,5-9 X 2,5-3,5 $\mu \mathrm{m}$.

Sobre Solanum lycopersicon L., su distribución geográfica es bastante amplia, ha sido citado en: Brunei, Holanda, India, Inglaterra, Malasia, Nueva Guinea, Nigeria, Papua, Polonia, Rodesia, Taití, Togo, Tonga.

Recolectado siempre sobre Solanum lycopersicon L. en El Prat de Llobregat (22-III85) y Seva (18-VI-85).

Phoma macrostoma Mont. Ann. Sci. Nat., ser 3 11:52 (1849).

Forma colonias de crecimiento rápido con micelio aéreo fieltroso o lanoso, con áreas blancas, grises u oliváceas; colonias de color rojo lívido que pasan a azul purpúreo al añadir $\mathrm{NaOH}$. Los conidios (5,5-10 X 2,5-3,5 $\mu \mathrm{m})$ de morfología muy variable son cilíndricos, elipsoides o piriformes, variablemente gutulados ocasionalmente presentan un septo.

Citado en especies de 41 géneros de hospedantes en Alemania, Chipre, Egipto, Etiopía, Holanda, Hong Kong, India, Kenia, Nepal, Nigeria, Nueva Zelanda, Pakistán, Sri Lanka, Suecia, Tanzania, Turquía y Venezuela.

Recolectada en Bellvei sobre Lactuca sativa L. (29-IV-88); en Castellet i La Gornal sobre Solanum tuberosum L. (29-IV-88); en Golmés sobre Prunus domestica L. (27-I-86) y en Malgrat de Mar sobre Raphanus sativus L. (14-III-88).

Phoma medicaginis var. pinodella (L.K. Jones) Boerema apud Boerema, Dorenbosch \& Leffring, Neth. J. Pl. Path 71:88 (1965).

Colonias extremadamente variables, a menudo forman diversos sectores con micelio 
aéreo fieltroso denso y oscuro o pálido y picnidios escasos y pequeños o bien micelio aéreo ralo con picnidios abundantes; formación de clamidósporas en cultivos jóvenes; en M.A. produce agregados cristalinos en el medio en forma de abanico. La tasa de crecimiento es mayor que en Phoma medicaginis var. medicaginis. Conidios rectos, cilíndricos o elipsoidales, de 7-10 × 2,5-4 $\mu \mathrm{m}$, a menudo con pequeñas gútulas y/o un septo transversal.

Citado sobre: Beta vulgaris, Casuarina sp., Coffea arabica, Galantus sp., Gossypium sp., Oryza sp., Petroselenium sativum, Phaseolus aureus, Pisum sativum, Pisum sp., Trifolium repens, Tr.pratense, Trifolium sp., Vicia faba, Vigna sinensis; Heno y Paja en Canadá, Holanda, India, Inglaterra, Nigeria, Nueva Zelanda y Tanzania.

Recolectado sobre Vicia faba L. en Celra (10-III-85).

Phoma pomorum Thüm., Fungi pomicoli: 105 (1879).

Colonias extremadamente variables, a menudo con sectores que presentan una abundante producción de picnidios y micelio aéreo pequeño o denso y fieltroso de color verde oliváceo, blanco, gris o negro. Conidios rectos o ligeramente curvados, cilíndricos o elipsoides, ocasionalmente gutulados, de 4,5-6 × 2-2,5 $\mu \mathrm{m}$. Forma clamidósporas aisladas y en agregados que recuerdan por su morfología y coloración los conidios de Alternaria.

Citado sobre: Arachis hypogaea, Capsicum sp., Ceonothus dentatus, Gossypium sp., Lithospermum sp., Malus sylvestris, Pinus caribaea, Poligonum tataricum, Prunus amygdalus, Pr. persica, Pyrus communis, Pyrus malus, Rhododendron sp., Salvinia modesta, Solanum tuberosum, Trichosantes dioica, y en el suelo en Holanda, India, Inglaterra, Libia, Nepal, Nigeria, Nueva Guinea, Papua, Sabah y Sri Lanka.

Recolectado en: Aitona sobre Prunus persica L. (Batsch.) (03-XII-84); Albatarrec sobre Prunus domestica L. (17-IX-87); Alcarras sobre Pyrus communis L. (10-XII-82); Alcoletge sobre Pyrus malus L. (13-V-84); Alguaire sobre Pyrus communis L. (03-II-88); Alpicat sobre Pyrus malus L. (12-II-88); L'Atmella del Vallés sobre Pinus pinea L. (18-X88); Arbucies sobre Pyrus malus L. (30-III-84); Arenys de Mar sobre Pinus pinaster Soland. (16-VIII-88); Artesa de Segre sobre Pyrus malus L. (10-VIII-87); Banyoles sobre Pinus halepensis Mill. (13-III-81); Barcelona sobre Pinus pinea L. (10-XI-87), Pinus sp. (14-III86), Prunus laurocerasus L. (30-I-89), Prunus subhirtella Miq. (16-VIII-84), Taxus baccata L. (29-V-87); Bascara sobre Albizzia julibrissin Durazz. (26-XI-87); Bellvis sobre Pinus halepensis Mill. (07-X-83); Benavent de Segrià sobre Pyrus malus L. (03-XII-84); Borrasa sobre Prunus dulcis (Miller) D.A. Webb (02-I-89); Bot sobre Prunus persica (L.) Batsch. (04VII-88); Brunyola sobre Pinus pinea L. (12-XII-86); Cambrils sobre Prunus persica $(\mathrm{L}$.$) Batsch.$ (15-IX-82); Camprodón sobre Pinus uncinata Ram. (17-VI-87); Cassà de la Selva sobre Pyrus communis L. var. conference (20-XII-84); Cervelló sobre Pinus halepensis Mill. (13-VII-88); Fontanilles sobre Pyrus communis L. (23-V-84); Fornells sobre Pyrus malus L. cv. early B1. (04-IV-84); Gandesa sobre Pinus halepensis Mill. (06-VII-84); Garriguella sobre Vitis vinifera L.cv. carinyena (28-V-85); Gavà sobre Pyrus malus L. (29-VI-88); Godall sobre Prunus persica (L.) Batsch. (18-V-84); Horta de St. Joan sobre Cydonia oblonga Miller (08-V-89); Lleida sobre Pyrus malus L. (120V-84) y (16-XII085), Pyrus communis L. cv. llimonera (16-XII84), Pyrus communis L. (15-IV-87); Llinars del Vallès sobre Pinus sp.; Moià sobre Pinus halepensis Mill. (26-V-84); Mollet del Vallès sobre Pinus sp. (26-V-87); Montroig del Camp sobre Prunus persica (L.) Batsch. (05-02-82); Mora d'Ebre sobre Prunus dulcis (Miller) D.A. Webb (14-IV-87); Olivella sobre Pinus halepensis Mill. (01-VIII-84); Palau Savardera sobre Prunus armeniaca L. (01-IV-86); Pals sobre Pinus pinea L. (17-XII-84); Peralada sobre Prunus dulcis (Miller) D.A. Webb (22-IV-85); La Pobla de Claramunt sobre Pinus halepensis Mill. 
(06-V-85); Premiá de Dalt sobre Pinus pinea L. (05-XII-81), Pinus sp. (11-X-88); Ripoll sobre Cydonia oblonga Miller (10-VI-88), Prunus persica (L.) Batsch. (10-VI-88); Rodonyà sobre Prunus dulcis (Miller) D.A. Webb (09-IV-86); Sta Fe del Montseny sobre Sequoia gigantea (Lindl.) Decne. (05-IV-88); St. Mateu de Bages sobre Pinus halepensis Mill. (16-IV-86); Seva sobre Pinus nigra Arnold (11-I-84); Tarragona sobre Pyrus malus L. (18-IX-82), Pinus halepensis Mill. (09-V-86); Tiana sobre Prunus armeniaca L. (25-X-82); Tornabous Prunus dulcis (Miller) D.A. Webb (29-05-86); La Torre de Claramunt sobre Pinus halepensis Mill. (06-V-85); Tortosa sobre Cedrus sp. (10-XI-88); Ulldecona sobre Prunus persica (L.) Batsch. (23-IV-87); El Vendrell sobre Pinus halepensis Mill. (17-11-87); Vilablareix sobre Pyrus communis L. (06-IX-82); Vilanova de Segrià sobre Prunus domestica L. (10-XI-84); Vilaseca i Salou sobre Pyrus communis L. (11-VI-82); Vilaverd sobre Corylus avellana L. (09IV-86).

Phoma tracheiphila (Petri) Kantachaveli \& Gikachvili.

Colonias con micelio aéreo denso de color gris bastante uniforme a blanco; formación de picnidios variable. Los conidios (4,5-6,5 x 1,5 $\mu \mathrm{m})$ cilíndricos, rectos o ligeramente curvados, no gutulados, se forman dentro de los picnidios sobre conidióforos cortos, tabicados y ramificados irregularmente o bien se forman en conidióforos desarrollados directamente sobre el miclio aéreo.

Citado sobre Citrus limon, C. medica, C. medica var. limon y Citrus sp. en Chipre, Irak, Italia, Turquía y Uganda.

Recolectado en Tortosa sobre Citrus aurantium L. (02-III-88) y Citrus nobilis Loureiro var. delic. (28-V-87).

Phoma tracheiphila forma specialis chrysantemi K.F. Baker, L.H. Davis, S. Wilhelm \& W.C. Snyder ha sido citada sobre Chrysanthemum morifolium, (Kirk, 1986).

\section{CONCLUSIONES}

La infección por especies del género Phoma en sus respectivos hospedantes se presenta prácticamente a lo largo de todo su ciclo vegetativo. Entre estos se incluyen tanto especies herbáceas (Solanum tuberosum L., Fragaria vesca $\mathrm{x}$ Fr. chiloensis, etc) como leñosas de porte arbustivo y arbóreo.

Son nuevas citas para la micoflora catalana Phoma cara Schulz., Ph. epicoccina Punithalingam. Tulloch et Leach, Ph. exigua Desm., Ph. exigua Desm. var. foveata (Foister) Boerema, Ph. glomerata (Cda.) Wollen et Hochapf., Ph. hedericola (Dur. et Mont.) Boerema, Ph. herbarum Wested., Ph. jolyana Pirozynski et Morgan-Jones, Ph. leveillei Boerema et Bollen, Ph. lycopersici Cke., Ph. acrostroma Mont., Ph. medicaginis var. pinodella (L.K. Jones) Boerema apud Boerema, Dorenbosch \& Leffring, Ph. pomorum Thüm. y Ph. tracheiphila (Petri) Kantachaveli \& Gikachvili. 


\section{BIBLIOGRAFÍA}

ELLIS, M.B. -1971- Dematiaceous Hyphomycetes Commomwealth Mycological Institute. Kew.

ELLIS, M.B. \& J.P. ELLIS -1985- Microfungi on Land Plants Croomttelm. London, Sidney. GONZALEZFRAGOSO, R. -1926-Botánica criptogámica agrícola. Espasa-Calpe. Barcelona. KIRK, P.M. -1986- Index of Fungi C.A.B. International Mycological Institute.

LOSA, M. -1948- Micromicetos del Pirineo Español. An. del Jardín Botánico de Madrid. T. VII (1947).

NADAL, M. \& A. MORET -1982- Espècies fitoparàsites de la Fam. Esferopsidàcies a Catalunya. Collectanea Botanica, vol. XIII. Barcelona.

SUTTON, B.C. -1980 The Coelomycetes Commonwealth Mycological Institute. Kew, Surrey, England.

(Aceptado para su publicación en Junio de 1.990)

Dirección de los autores: Departamento de Biología Vegetal. Fitopatología. Facultad de Biología. Universidad de Barcelona. 